\title{
Mathematical modeling of infectious disease transmission in macroalgae
}

\author{
Artorn Nokkaew' ${ }^{1}$, Charin Modchang ${ }^{2,3}$, Somkid Amornsamankul ${ }^{3,4}$, Yongwimon Lenbury ${ }^{3,4}$, \\ Busayamas Pimpunchat ${ }^{5}$ and Wannapong Triampo $2,3^{*}$
}

\author{
"Correspondence: \\ wtriampo@gmail.com \\ ${ }^{2}$ Department of Physics, Faculty of \\ Science, Mahidol University, \\ Bangkok, 10400, Thailand \\ ${ }^{3}$ Centre of Excellence in \\ Mathematics, CHE, 328, Si Ayutthaya \\ Road, Bangkok, 10400, Thailand \\ Full list of author information is \\ available at the end of the article
}

\begin{abstract}
Understanding the infectious diseases outbreak of algae can provide significant knowledge for disease control intervention and/or prevention. We consider here a disease caused by highly pathogenic organisms that can result in the death of algae. Even though a great deal of understanding about diseases of algae has been reached, studies concerning effects of the outbreak at the population level are still rare. For this reason, we computationally model an outbreak in the algae reservoir or container systems consisting of several patches or clusters of algae being infected with a contagious infectious disease. We computationally investigate the systems as well as make some predictions via the deterministic SEIR epidemic model. We consider the factors that could affect the spread of the disease including the number of patches, the size of initial infected population, the distance between patches or spatial range, and the basic reproduction number $\left(R_{0}\right)$. The results provide some information that may be beneficial to algae disease control, intervention or prevention.
\end{abstract}

Keywords: outbreak; epidemic; infectious disease; algae; SEIR model; mathematical modeling

\section{Introduction}

Algae are aquatic primitive multicellular photosynthetic plant species that play an essential role in aquatic ecosystems. They can come in many forms and colors. Algae can be generally characterized based on their photosynthetic pigments and combinations thereof: Cyanophyta, blue-green algae; Rhodophyta, red algae; Chrysophyceae, golden algae; Phaephyceae, brown algae; Chlorophyta, green algae [1]. They range in size from tiny, $1 \mathrm{mi}-$ crometer in diameter, called microalgae, to giant kelps which can reach 60 meters in length, called macroalgae [2].

The macroalgae can be utilized as a crop [3]. Remarkably, it does not require any land or fertilizer. For farming purposes, it is not only used for direct consumption [4] in a number of ways such as human food, fish food, fertilizer, skin care, and biofuel, but also it can help to improve the environment. Like terrestrial forests, macroalgae forests (such as kelp forests) provide an extensive ecosystem for many organisms from the sea floor to the ocean surface. However, the increasing use of macroalgae as crop species for commercial purposes requires a good system and process for both cultivation and disease control.

Similar to any other living organisms, algae are plagued by diseases caused by fungi, bacteria or viruses. Here parasitism is mainly focused on. Parasitism is one of the common

(c) The Author(s) 2017. This article is distributed under the terms of the Creative Commons Attribution 4.0 International License (http://creativecommons.org/licenses/by/4.0/), which permits unrestricted use, distribution, and reproduction in any medium, provided you give appropriate credit to the original author(s) and the source, provide a link to the Creative Commons license, and indicate if changes were made. 
ecological interactions with algae. They represent a strong forcing factor for evolutionary and ecological processes, e.g., population dynamics, species successions, competition for resources, species diversification, and energy and gene flows [5]. Among their parasites, fungi are the most dominant ones [6]. Few attempts have been made to include parasites in the food web dynamics of aquatic systems [7]. As aquaculture continues to rise worldwide, pathogens of algae are becoming a significant economic burden. The filamentous green alga, Chaetomorpha media, from the western and eastern coasts of the Indian peninsula showed infection by an oomycetous fungus Pontisma lagenidioides. The infected cells appear brownish and the infection spreads from the tip downwards of the algal filament on incubation in seawater in the laboratory $[8,9]$.

In particular, infectious disease outbreak and control could become a huge problem for production management. Thus, in this work, a possible infectious disease outbreak or epidemic is our main focus. Even though the pathology aspect is quite well understood in the most part, how macroalgae respond to pathogens and how the disease can spread at the population level is not well known [10-12].

So far, to the best of our knowledge, there has been no publication on mathematical modeling studies about infectious disease outbreak or epidemics involving macroalgae. Since the epidemics could be extremely costly to farming, it is important to learn as much as possible how to prevent, control, or initiate an intervention, when it happens. Typically, there are many risk factors driving the emergence of the epidemic including population density, degree of transmission, degree of contagion, contact nature, water condition, and climate and so on $[13,14]$.

In this work, we apply a traditional susceptible-exposed-infectious-recovered (SEIR) model [13] to study the macroalgae system. Both time and spatial considerations were conducted. Computational results and analyses were given. Interpretation and connection between real world and model world were carefully done.

\section{Methods}

We develop a spatial compartmental model based on the traditional susceptible-exposedinfectious-recovered (SEIR) equations, where each patch of algae population is represented by a spatial compartment. $S_{i}, E_{i}, I_{i}$, and $R_{i}$ representing the numbers of susceptible (capable of becoming infected), exposed (latently infected and incapable of transmitting infection), infectious (infectious individuals capable of transmission), and removed or dead individuals in the $i$ th spatial compartment, respectively. Based on the model described above and the compartmentalized structure seen in Figure 1, the law of mass action leads us to the following set of coupled ordinary differential equations:

$$
\begin{aligned}
& \frac{d S_{i}}{d t}=-S_{i} \sum_{j=1}^{n} \beta_{i j} I_{j}, \\
& \frac{d E_{i}}{d t}=S_{i} \sum_{j=1}^{n} \beta_{i j} I_{j}-\sigma E_{i}, \\
& \frac{d I_{i}}{d t}=\sigma E_{i}-\gamma I_{i}, \\
& \frac{d R_{i}}{d t}=\gamma I_{i},
\end{aligned}
$$


Figure 1 The spatial compartment of macroalgae system. (A) A conceptual model of infectious disease transmission in the real world. (B) The representation of the transmission in the mathematical form. (C) Model structure. $S_{i}, E_{i}, l_{i}$, and $R_{i}$ represent the number of susceptible, exposed, infectious, and recovered algae in theith patch, respectively. The transition rate for each state is shown near the transition arrow.

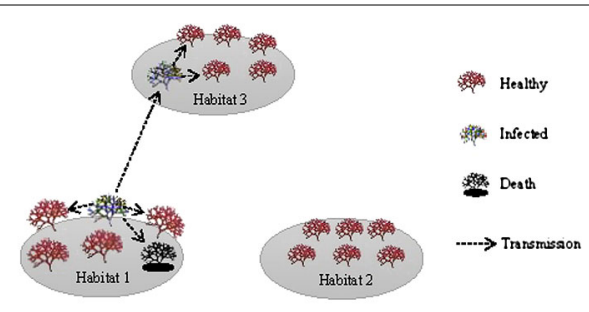

(A)

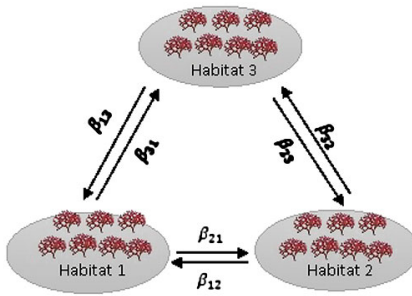

(B)

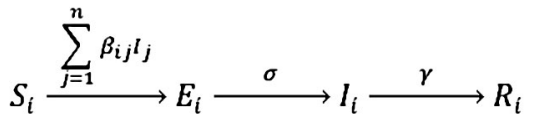

(C)

Table 1 Parameter values used in the simulations

\begin{tabular}{lc}
\hline Parameter & Value \\
\hline Basic reproduction number $\left(R_{0}\right)$ & 1.4 or 2 \\
Distance scaling factor $\left(r_{0}\right)$ & $30 \mathrm{~m}$ \\
Incubation period $(1 / \sigma)$ & 2 day \\
Infectious period $(1 / \gamma)$ & 5 day \\
Initial infectious individuals & $1-28 \%$ \\
Total number of algae individuals & 900 \\
Number of clusters & $2-6$ \\
Algae population of each cluster & $150-450$ \\
Area of each cluster & $1,000 \mathrm{~m}^{2}$ \\
Distance between clusters & $30-600 \mathrm{~m}$ \\
Time step & $10^{-3}$ day \\
\hline
\end{tabular}

where $i=1,2, \ldots, n$ and $n$ is the number of algae patches with the conservation constraint, $S_{i}+E_{i}+I_{i}+R_{i}=N_{i}$ where $N_{i}$ is the total number of algae in the $i$ th compartment (see the parameter values in Table 1 ).

Susceptible algae $S_{i}$ are infected at a rate of $\sum_{j=1}^{n} \beta_{i j} I_{j}$, where $\beta_{i j}$ is the transmission rate from the $j$ th patch to the $i$ th patch. The summation is taken over all of the compartments that can spread the infection to theith patch. The infected individuals $\left(E_{i}\right)$ incubate the infection for a mean duration of $1 / \sigma$. After passing through the exposed state, infected individuals become infectious $\left(I_{i}\right)$ with the mean duration of the contagious stage of $1 / \gamma$.

It should be noted that the dynamics of an epidemic here is assumed to be much faster than the dynamics of natural birth and death (vital dynamics), therefore, birth and death are omitted in the model. In other words the time scale of the epidemic dynamics of algal system is much faster than that of vital dynamics. Or the birth rate and death rate of algae is considerably much less than the incubation and infectious period of the disease. As far as our current model is concerned, it would be more realistic if the life span of algae is 
relatively long enough compared to the infectious period. It was estimated that the life span of the large brown attached alga, Macrocystis pyrifera, a member of a widespread genus, could be several months [15].

In epidemiology, the basic reproduction number, $R_{0}$, is defined as the average number of secondary cases arising from an average primary case in an entirely susceptible population. For the homogeneous system, where there is only one patch of algae, hence $\beta_{i j}=\beta$ and

$$
R_{0}=\frac{\beta}{\gamma} .
$$

In this work, we also incorporated the environmental spatial aspect into our model. In this case the transmission rate from the $j$ th patch to the $i$ th patch $\left(\beta_{i j}\right)$ is assumed to be

$$
\beta_{i j}=\kappa \frac{\rho_{i} \rho_{j}}{1+\left(r_{i j} / r_{0}\right)^{2}},
$$

where $\rho_{i}$ and $\rho_{j}$ are algae density in habitat $i$ and $j$, respectively, $r_{i j}$ is the distance between habitats $i$ and $j, r_{0}$ is a distance scaling factor, and $\kappa$ is a constant [16]. The constant $\kappa$ can be obtained by using the following expression $[17,18]$ :

$$
R_{0}=\max \left(\text { eigenvalue }\left(\frac{\left[\beta_{i j}\right]}{\gamma}\right)\right) \text {. }
$$

Unless stated otherwise, the parameter values used in the simulation are summarized in Table 1 . The model equations are numerically solved using the explicit Euler method. We numerically solved the model equations by using the explicit Euler method in the MATLAB software. All simulations were run with the time step of $10^{-3}$ day. The explicit Euler method was employed because of its simplicity. Although the Euler method has more local truncation error than other more advanced methods (e.g., RK4), we actually can reduce the truncation error by decreasing the time step. We have checked that using the Euler method with time step of $10^{-3}$ day does not show any noticeable numerical error and using smaller time step does not significantly improve the accuracy (data not shown). In addition, in our system there are two different spatial scales of the disease transmission. On the small scale, the disease spreads in a well-mixed patch of algae. Since we assumed a homogeneous mixing of algae on this scale, we believe that the dynamics of the disease transmission in a 2D- or a 3D-patch would be the same (given the same $R_{0}$ ). On a large scale, it is reasonable to assume that the transmission rate between any two patches depends only on the distance between those patches, not the actually locations. Hence on this scale, the problem is virtually a one-dimensional problem, in the sense that the transmission rate depends only on $r_{i j}$. We therefore believe that the proposed model should be able to describe the dynamics of infectious disease transmission in algae.

\section{Results and discussion}

In this study, we quantitatively investigated the factors that cause the damage of algae farm due to an epidemic of an infectious disease. The factors studied in this investigation include $R_{0}$, the number of patches (density of a planting patch), the distance between the patches and the number of initial infectious individuals. 


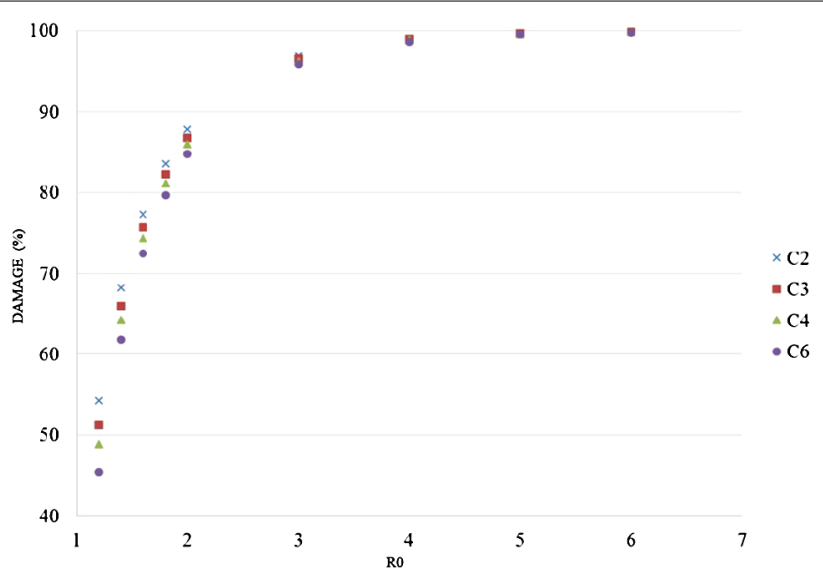

Figure 2 Plots show relationships between $R_{0}$ and the number of patches ranging from two to six patches (C2-C6) with the percentage of damage.

To investigate the influence of $R_{0}$ and the number of patches on the loss in algae production or damage or the number of recovered individuals at the equilibrium of the model, we vary the value of $R_{0}$ and the number of patches. In our system, the damage is highly sensitive to the level of $R_{0}$. In addition, the damage is more sensitive to the number of patches where $R_{0}$ is small (see Figure 2). At $R_{0}$ of 1 to 2, the smaller number of patches, the higher the percentage of damage. The sensitivity reduces when $R_{0}$ gets higher. When $R_{0}$ is less than 1.5 , the damage increases by more than $10 \%$. The percentage of damage decreases when $R_{0}$ increases. This trend is found to be similar for all numbers of patches used in this study.

From further investigation, it is found that the number of patches is not the only factor that influences the production loss, but the distance between the patches also plays a role. The distance has been varied to 1, 2, 5, 10 and 20 times $r_{0}$. Two different behaviors can be observed (see Figure 3). For the distance of $r_{0}$ or twice $r_{0}$, the percentage of damage decreases when the number of patches increases. However, the percentage of damage is constant for the distance larger than $5 r_{0}$. This behavior is similar for a disease of $R_{0}$ of 1.4 and 2.0.

Considering the effect of patch numbers and the time of the peak of the epidemic, unlike the percentage of the damage, the day of the maximum of the epidemic is sensitive to $R_{0}$. Different behaviors were found for $R_{0}$ of 1.4 and 2.0. For $R_{0}$ of 1.4, the data of the peak varied. For $R_{0}$ of 2.0, three patterns were found. For $10 r_{0}$ and $20 r_{0}$, the highest peak is slightly decreased with increase of the number of clusters (see Figure 3). For $1 r_{0}$ and $2 r_{0}$, the period of the peak is slightly longer as the number of patches increases. For $5 r_{0}$, the period of the peak is close to constant for $R_{0}$ of 1.4 and 2.0. Note that the maximum number of infectious (at the peak) is different for different distances. Based on our system, a longer distance yields a lower maximum number. Computationally, we performed simulation and measured the following quantities, namely statistical hypothesis testing for investigation between the number of patches $\left(c_{i}, i=2,3,4,5\right.$ and 6$)$ on the loss or damage and the distance among patches such that $1 r_{0}, 2 r_{0}, 5 r_{0}, 10 r_{0}$ and $20 r_{0}$, respectively. We can test whether or not the number of patches and the distance between patches differ among groups and conduct the $F$-test at the $\alpha=0.05$ significant level. As a result, we rejected H0, 

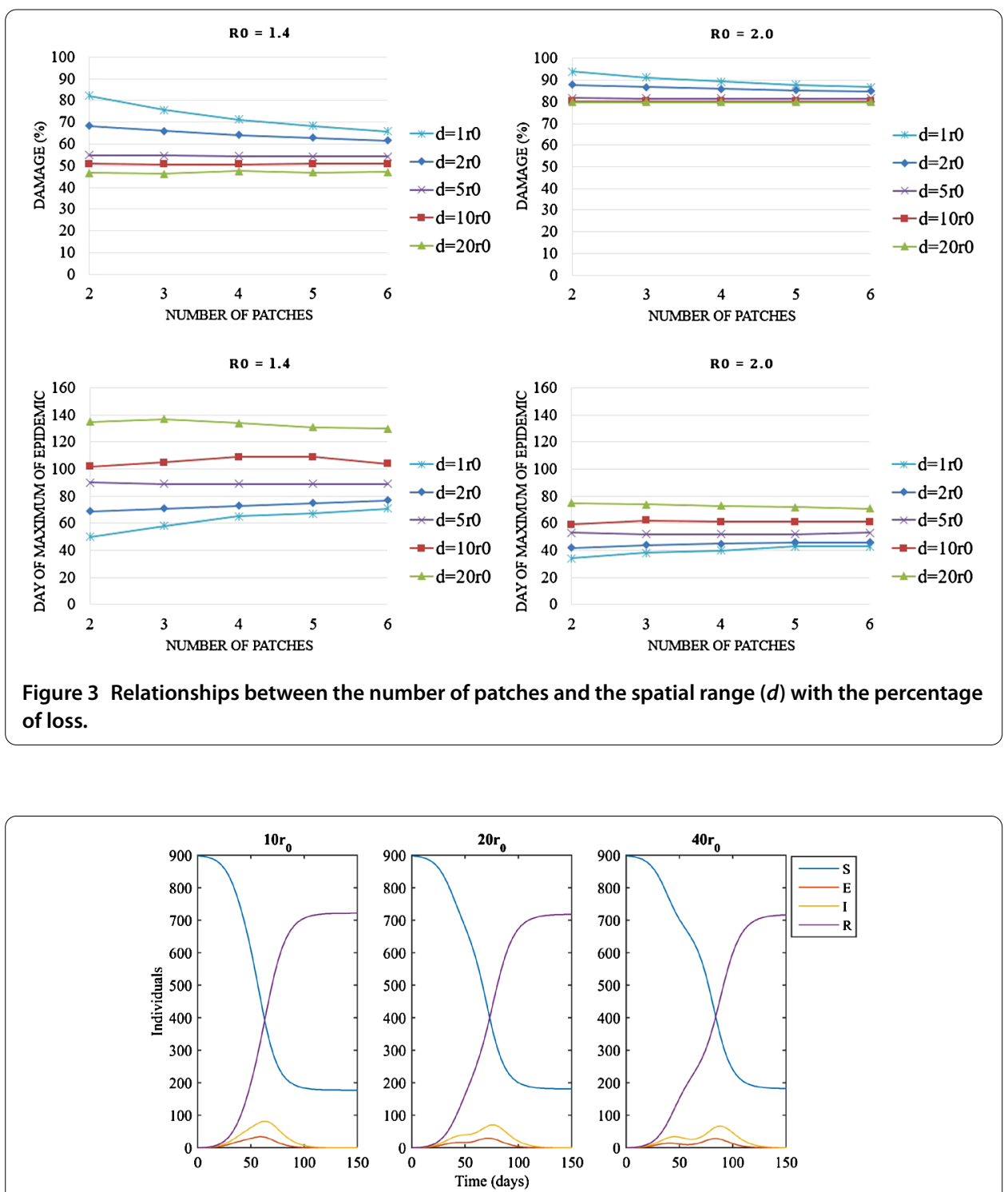

Figure 4 Dynamics of populations with varying number of patches $\left(R_{0}=2, C=3\right)$.

and concluded that a difference existed between the various values of $R_{0}$. There was no difference with regard to the number of patches.

In Figure 4, the longer time required to reach the peak of the epidemic due to the distance can be explained by the emergence of two peaks of the infections. As a consequence, it postpones the highest peak of the infectious which we consider to be one of the main factors that affects the damage of production. We have also found that as the distance increases, the first peak becomes clearer. We have tested this point by changing the number of patches in our system to six and run the simulations again (data not shown). We found that there are still two peaks (not five peaks) even when $c=6$. This result does make sense because in our model all patches are connected with an equal separating distance. The first peak, therefore, corresponds to the epidemic in the initially infected cluster and the second peak accounts for the epidemic of the remaining five cluster. 


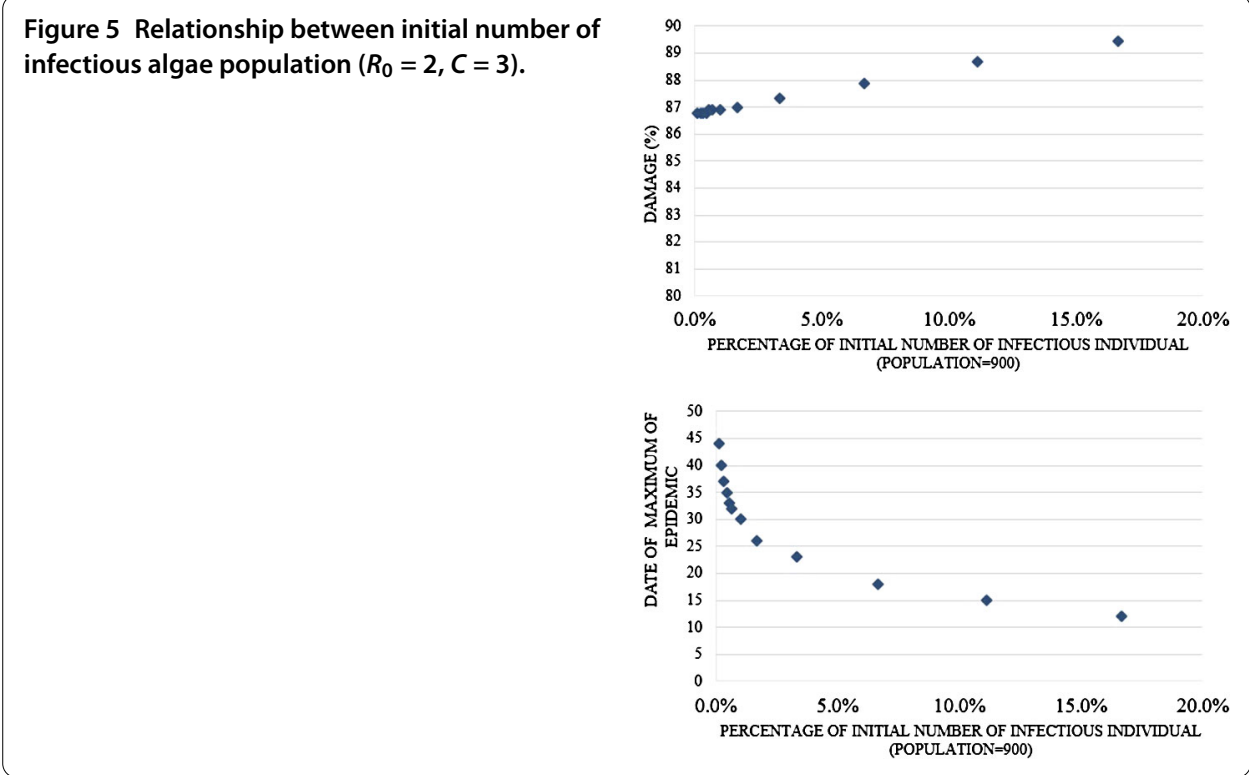

The last factor that we investigated in this study is the number of initial infectious algae populations. It may be expected that when the initial number of infectious population increases, the loss should also increase. This is confirmed by our model as shown in Figure 5 . For a certain small number of initial infectious algae populations, the change slightly affects the loss. The effect increases in degree as the initial infectious algae population becomes greater than 10 (see Figure 5). Note that $R_{0}$ for this investigation is 2 and the number of patches is three. Two different behaviors have also been observed. Of course, it would be evidently clearer if one had measured the number of infectious individuals due to the epidemics in comparison with that due to the initial number of infectious algal individuals. Unfortunately, because of the time constraint we cannot make it for the time being. We, however, believe this could be the case as we consider if we would be concerned with large scale imports from corporations starting macroalgae farms. Our observation could be useful in the real-world system of cultivation of macroalgae. At this state of research it is based on the modeling and computational aspects. It remains to be seen whether this study will be useful enough to apply to real-world problems.

\section{Conclusions}

In this work, we applied the SEIR epidemics model to study the infectious disease spreading in algae population. Our SEIR-based model suggests that the value of $R_{0}$ plays a significant role in the epidemic dynamics of algae system. Our results are considerably consistent with the general theory. Due to the fact that $R_{0}$ is the number of cases that one case generates on average over the course of its infectious period, the larger the value of $R_{0}$, the more the infection can spread, and the harder it is to control the epidemic. When we compartmentalized the whole population into smaller compartments or clusters, it results in a lower algae population density and consequently a lower contact rate as well. However, in this work we used the transmission rule based on the modified gravity disease transmission model. It is apparent from the disease transmission rate that the distance between habitats plays a crucial role on the spread of the disease. Lastly, the number of clusters or 
habitats (for a given total population) is found to mitigate the epidemics when the number is increased.

\author{
Acknowledgements \\ This research project is supported by the Centre of Excellence in Mathematics, the Commission on Higher Education, \\ Thailand.
}

\title{
Funding
}

This research project is supported by the Centre of Excellence in Mathematics, the Commission on Higher Education, Thailand. The sponsors of the study had no role in the study design; collection, analysis and interpretation of data; the writing of the manuscript; and the decision to submit the manuscript for publication.

\section{Competing interests}

The authors declare that there are no conflicts of interest.

\section{Authors' contributions}

AN, CM, and WT designed, did experiments, analyzed results, and wrote the manuscript. SA, BP, and YL analyzed results, and wrote the manuscript. All authors read and approved the final manuscript.

\section{Author details}

${ }^{1}$ Institute for Innovative Learning, Mahidol University, Nakorn Pathom, 73170, Thailand. ${ }^{2}$ Department of Physics, Faculty of Science, Mahidol University, Bangkok, 10400, Thailand. ${ }^{3}$ Centre of Excellence in Mathematics, CHE, 328, Si Ayutthaya Road, Bangkok, 10400, Thailand. ${ }^{4}$ Department of Mathematics, Faculty of Science, Mahidol University, Bangkok, 10400, Thailand. ${ }^{5}$ Department of Mathematics, Faculty of Science, King Mongkut's Institute of Technology Ladkrabang, Bangkok, 10520, Thailand.

\section{Publisher's Note}

Springer Nature remains neutral with regard to jurisdictional claims in published maps and institutional affiliations.

Received: 1 February 2017 Accepted: 26 August 2017 Published online: 15 September 2017

\section{References}

1. Hoek, C, Mann, D, Jahns, HM: Algae: An Introduction to Phycology. Cambridge University press, Cambridge (1995)

2. Lewin, RA: Encyclopedia Britannica. (2014) http://global.britannica.com/EBchecked/topic/14828/algae. Accessed 28 February 2014

3. Rigney, JA: Chemical Investigations of Chondrus Crispus Progress Report. 87 (1971)

4. FAO: The State of World Fisheries and Aquaculture (2014)

5. Hudson, PJ, Dobson, AP, Lafferty, KD: Is a healthy ecosystem one that is rich in parasites? Trends Ecol. Evol. 21(7), 381-385 (2006)

6. Raghukumar, C: Zoosporic fungal parasites of marine biota. In: Dayal, R (ed.) Advances in Zoosporic Fungi, pp. 61-83. M.D. Publications Pvt. Ltd., New Delhi (1996)

7. Amundsen, PA, Lafferty, KD, Knudsen, R, Primicerio, R, Klemetsen, A, Kuris, AM: Food web topology and parasites in the pelagic zone of a subarctic lake. J. Anim. Ecol. 78(3), 563-572 (2009)

8. Raghukumar, C: Fungal pathogens of the green alga Chaetomorpha media. Dis. Aquat. Org. 3, 147-150 (1987)

9. Raghukumar, C, Chandramohan, D: Post-infectional changes in the green alga Chaetomorpha media infected by a fungus. Bot. Mar. 31, 311-315 (1988)

10. Andrews, JH: The pathology of marine algae. Biol. Rev. 51, 211-253 (1976)

11. Andrews, JH: Pathology of seaweeds: current status and future prospects. Experientia 35, 429-450 (1979)

12. Gachon, CMM, Sime-Ngando, T, Strittmatter, M, Chambouvet, A, Kim, GH: Algae diseases: spotlight on a black box. Trends Plant Sci. 15, 633-640 (2010)

13. Anderson, RM, May, RM, Anderson, B: Infectious Diseases of Humans: Dynamics and Control. Oxford University Press, London (1992)

14. Murray, JD: Mathematical Biology: I. An Introduction, 3rd edn. Springer, Berlin (2002)

15. North, WJ: Life-span of the fronds of the giant kelp, Macrocystis pyrifera. Nature 190, 1214-1215 (1961)

16. Sornbundit, K, Triampo, W, Modchang, C: Mathematical modeling of diphtheria transmission in Thailand. Comput. Biol. Med. 87, 162-168 (2017)

17. Diekmann, O, Heesterbeek, JAP: Mathematical Epidemiology of Infectious Diseases: Model Building, Analysis, and Interpretation. Wiley, New York (2000)

18. Diekmann, O, Heesterbeek, JAP, Roberts, MG: The construction of next-generation matrices for compartmental epidemic models. J. R. Soc. Interface 7(47), 873-885 (2010) 